\title{
ALTA CAPACIDAD Y GÉNERO: LA AUTOESTIMA COMO FACTOR INFLUYENTE EN LAS DIFERENCIAS ENTRE SEXOS
}

\author{
Ramón García Perales \\ Inmaculada Canuto González \\ Antonio Cebrián Martínez \\ Universidad de Castilla La Mancha
}

RESUMEN: Los resultados obtenidos en el diagnóstico de altas capacidades son significativamente menores en niñas que en niños; sin embargo, no se encuentran estudios concluyentes que confirmen diferencias en la inteligencia en función del sexo. La literatura publicada sitúa los estereotipos de género y la autoestima como variables fundamentales en la brecha existente. Razonadamente, hemos realizado una investigación cuantitativa de la autoestima en 118 sujetos de $4^{\circ}$, $5^{\circ}$ y $6^{\circ}$ de Educación Primaria, comparando después los resultados en función de distintas variables moduladoras. Se ha utilizado la Escala de Autoestima de Rosenberg. Los resultados confirman que sí existen diferencias en la autoestima en función del sexo, siendo las niñas las que presentan niveles más bajos. El rendimiento académico es otra variable que correlaciona positivamente con la autoestima. No se han hallado resultados significativos que relacionen el constructo evaluado con la edad, el curso, las altas capacidades diagnosticadas o el tipo de centro de los sujetos. Las conclusiones se centran en la demanda de nuevas investigaciones que analicen la posible vinculación de la discriminación de la mujer con su no adecuada autoestima, centrándonos en las mujeres con altas capacidades.

PALABRAS CLAVE: Altas capacidades, género, autoestima, estereotipos, igualdad de oportunidades.

\section{HIGH ABILITIES AND GENDER: A SELF-ESTEEM AS AN INFLUENTIAL FACTOR IN THE DIFFERENCES BETWEEN SEXES}

\footnotetext{
ABSTRACT: The results obtained in the diagnosis of high abilities are lower in girls than in boys; however, there are not conclusive studies
} 
that confirm differences in intelligence depending on sex. The published literature places gender stereotypes and self-esteem as fundamental variables in this existing gap. Reasonably, we have carried out a quantitative investigation of self-esteem in 118 subjects of 4 th, 5th and 6th grade of Primary Education, comparing the results according to different modulating variables. We have used the Rosenberg Self-Esteem Scale. The results confirm that there are differences in self-esteem according to sex, with girls having the lowest levels. Academic results are another variable that correlates positively with self-esteem. No significant results have been found that relate the evaluated constructs with age, course, high abilities diagnosed or the subjects type of center. The conclusions focus on the demand for new research that analyzes the possible link of discrimination of women with their lack of self-esteem, focusing on women with high abilities.

KEYWORDS: High abilities, gender, self-esteem, stereotypes, equal opportunities.

Recibido: $17 / 12 / 2918$

Aceptado: 14/06/2019

Correspondencia: Ramón García Perales, Universidad de Castilla La Mancha, Plaza de la Universidad 3, 02071 Albacete. Email: Ramon.GarciaPerales@uclm.es.

\section{INTRODUCCIÓN}

Las altas capacidades intelectuales siguen siendo, aún hoy, la asignatura pendiente de la atención a la diversidad y del sistema educativo español. La complejidad y heterogeneidad que encierra esta condición da lugar a una problemática en cuanto a su detección e intervención educativa que merma el desarrollo de las capacidades de muchos alumnos y alumnas, perdiéndose así un excelente potencial muy valioso para nuestra sociedad. Además, la carencia de respuestas educativas individualizadas puede derivar en graves problemas socioemocionales y educativos como depresión infantil, ansiedad, problemas conductuales, aislamiento social, dificultades de aprendizaje y fracaso escolar.

Y si a este escenario se le añade la variable género, la situación se complica todavía más, resultando la niña altamente capaz, la gran perjudicada. Así lo indican los últimos datos publicados, para enseñanzas no universitarias, por el Ministerio de Educación Cultura y Deporte en el curso 2016/2017, tomando en consideración el número de alumnos diagnosticados para cada Comunidad Autónoma, junto al sumatorio nacional, y para la variable género, recogidos en la Tabla 1. 
Tabla 1. Alta capacidad en España según el género en el curso 2016/2017

\begin{tabular}{|c|c|c|c|c|c|}
\hline \multirow{2}{*}{$\begin{array}{c}\text { CC. AA. } \\
\text { Curso 2016/2017 }\end{array}$} & \multirow{2}{*}{$\begin{array}{l}\mathrm{N} \cdot{ }^{\circ} \text { total de } \\
\text { alumnos con } \\
\text { alta capacidad }\end{array}$} & \multicolumn{2}{|c|}{ Hombres } & \multicolumn{2}{|c|}{ Mujeres } \\
\hline & & $\mathbf{n}$ & $\%$ & $\mathbf{n}$ & $\%$ \\
\hline Andalucía & 11.582 & 5942 & 51,30 & 5.640 & 48,70 \\
\hline Aragón & 182 & 126 & 69,23 & 56 & 30,77 \\
\hline Principado de Asturias & 804 & 484 & 60,20 & 320 & 39,80 \\
\hline Illes Balears & 831 & 473 & 56,92 & 358 & 43,08 \\
\hline Canarias & 2.122 & 1205 & 56,79 & 917 & 43,21 \\
\hline Cantabria & 128 & 77 & 60,16 & 51 & 39,84 \\
\hline Castilla y León & 638 & 440 & 68,97 & 198 & 31,03 \\
\hline Castilla-La Mancha & 411 & 267 & 64,96 & 144 & 35,04 \\
\hline Cataluña & 417 & 235 & 56,35 & 182 & 43,65 \\
\hline Comunitat Valenciana & 1.063 & 654 & 61,52 & 409 & 38,48 \\
\hline Extremadura & 266 & 178 & 66,92 & 88 & 33,08 \\
\hline Galicia & 1.590 & 991 & 62,33 & 599 & 37,67 \\
\hline Comunidad de Madrid & 2.190 & 1426 & 65,11 & 764 & 34,89 \\
\hline Región de Murcia & 3.698 & 1760 & 47,59 & 1.938 & 52,41 \\
\hline Comunidad Foral de Navarra & 399 & 236 & 59,15 & 163 & 40,85 \\
\hline País Vasco & 536 & 353 & 65,86 & 183 & 34,14 \\
\hline Rioja & 274 & 182 & 66,42 & 92 & 33,58 \\
\hline Ceuta & 2 & 1 & 50,00 & 1 & 50,00 \\
\hline Melilla & 0 & 0 & 0,00 & 0 & 0,00 \\
\hline España & 27.133 & 15030 & 55,39 & 12.103 & 44,61 \\
\hline
\end{tabular}

Fuente: Elaboración propia a partir de datos del Ministerio de Educación Cultura y Deporte-MECD- (2018).

Sin embargo, estos datos relativos a la menor prevalencia de mujeres entre los alumnos con altas capacidades, no se encuentran respaldados por la evidencia científica, pues ningún estudio demuestra de manera concluyente que existan diferencias biológicas o cognitivas entre niños y niñas que puedan explicar esta brecha existente en el diagnóstico de altas capacidades. Durante los siglos XIX y principios del XX la investigación en torno al tema ha sido muy limitada, puesto que históricamente se ha considerado a la mujer inferior al hombre en todos los aspectos, incluido en lo que a capacidad intelectual se refiere. Pero, en la actualidad, sí que encontramos distintas investigaciones que han tratado de analizar las diferencias cognitivas que pudieran existir entre niños y niñas para intentar dar respuesta a la situación que se plantea (Baeza y Lamadrid, 2018; Bian, Leslie, y Cimpian, 2017; Choi y Calero, 2013).

Garaigordobil y Amigo (2010) Ilevaron a cabo un estudio con la intención, entre otras, de explorar diferencias de género en capacidades cognitivas durante la infancia temprana. Estas investigadoras no encontraron diferencias de género en aspectos intelectivos ni en la capacidad de pensamiento abstracto entre niños y niñas, aunque citan una serie de investigaciones con resultados discrepantes que sí evidenciaron diferencias entre sexo e inteligencia, pero en edades más tardías, a partir de los siete 
u ocho años. Sin embargo, más que diferencias en aspectos cognitivos, los resultados de esos estudios se refieren más bien a diferencias en el desempeño de distintas áreas y tareas, como pueden ser la habilidad matemática, el desarrollo del lenguaje, la inteligencia musical, el desempeño social, etc. Concluyen Garaigordobil y Amigo que se puede afirmar que no existen diferencias cognitivas ni de rendimiento entre los 6 y los 14 años, sino que éstas aparecerían en la adolescencia y serían más notables en la edad adulta (2010).

Por otro lado, Campo (2016) hace un análisis pormenorizado de los estudios sobre inteligencia y sexo desde que estos se iniciasen a principios del siglo XX. De entrada, y como decíamos, muchas de estas investigaciones situaban claramente a la mujer como inferior al hombre, si bien después se fue constatando que las diferencias entre niños y niñas se daban en función de las aptitudes, puesto que las niñas superan a los niños en capacidad verbal y los niños puntúan más alto en los campos matemático y espacial (Elices et al., 1990; Instituto Nacional de Evaluación Educativa -INEE-, 2013; Jiménez et al., 2010; Llor et al., 2012; MECD, 2016a, 2016b; Organización para la Cooperación y el Desarrollo Económico-OCDE-, 2014; Pasarin et al., 2004). Y, así, Campo sintetiza su indagación afirmando que "las diferencias más claras entre los sexos aparecen en el rendimiento ante determinadas tareas y no tanto en el Cl total" (2016, p. 50), concluyendo que las diferencias significativas se manifiestan en mayor medida cuando aumenta la edad.

En este sentido, apunta también el trabajo de Pérez y Domínguez (2000) en el que se estudió el porcentaje de alumnos con altas capacidades intelectuales en la Comunidad de Madrid en función del sexo, que determinó que no existían diferencias entre los 3 y los 5 años, mientras que de los 6 a los 12 años encontraron un 48\% de niñas frente a un $52 \%$ de niños con altas capacidades, y observando que de los 13 a los 17 la brecha amentaba, con un $27 \%$ frente a un $73 \%$, constatando que disminuye considerablemente la proporción de niñas con altas capacidades a medida que llega la adolescencia.

De esta forma, debería de analizarse los factores que inciden en estas diferencias mostradas con el análisis de estos estudios y de los datos que aportan. Así, podría inducirse que las diferencias en el nivel cognitivo irían asociadas, más que a una cuestión de sexo biológico, a una cuestión del rol de género que el niño y la niña van a adoptando conforme van creciendo en sociedad, cuestión que se expone ampliamente en diferentes investigaciones (Colás y Villaciervos, 2007; García et al., 2013; Saavedra et al., 2013; Suberviola, 2012). Algunas se exponen a continuación.

De este modo Peña del Agua (2002) sostiene que el rol de género femenino se interpone en el desarrollo del talento de las niñas. Lo mismo que Hollingworth (citada en Kreger, 1999), que afirmaba que el número limitado de mujeres reconocidas como excepcionales era debido a casuísticas culturales y no biológicas, pues defendía que lo que una chica puede llegar a conseguir depende en parte de sus dotes congénitas, pero que el éxito que realmente cosecha es consecuencia del ambiente. GonzálezPalencia y Jiménez (2016, pp. 745-746) también defienden que no se debe asumir que los varones tienen mayor capacidad que las mujeres, si no que la "hipótesis del 
entrenamiento y la socialización según el género" sería la que toma mayor fuerza en esta situación.

La misma línea argumental sigue el informe de la OCDE denominado The $A B C$ of Gender Equality in Education: Aptitude, Behaviour, Confidence, que analizaba las variables educación y género con datos desde 2000 a 2012. En el mismo se señala que "la brecha de género en rendimiento académico no se encuentra determinada por diferencias innatas de capacidad" (OCDE, 2012, p. 3). Según este organismo internacional, bajo condiciones semejantes, chicos y chicas pueden alcanzar mismos niveles de desempeño. Pese a esto, las diferencias entre géneros son evidentes ya que los chicos rinden menos y tienden a dejar los estudios más tempranamente. En contraposición, después los hombres tienen más éxito en el mundo laboral, con remuneraciones más elevadas y mayor representación en puestos directivos. Según el Informe Español de la OCDE teniendo en consideración el programa Programme for International Student Assessment (PISA) en su edición de 2015 (OCDE, 2016), se siguen observando estas diferencias de rendimiento según el género. En lectura, las chicas puntúan significativamente más alto que los chicos en todos los países analizados, mientras que en matemáticas y ciencias esta situación cambia a favor de los chicos.

Como vemos, la realidad es que las alumnas igualan e incluso superan en algunas destrezas a los alumnos durante la Educación Primaria y parte de la Secundaria (OCDE, 2012, 2016). Sin embargo, parece ser que es en el momento del inicio de la adolescencia, estudios de Educación Secundaria, cuando se abre una brecha entre hombres y mujeres en lo que a capacidades, aptitudes y éxito personal y académico se refiere. ¿Por qué sucede esto? Pasamos a continuación a profundizar en los motivos de estas discrepancias entre sexos.

Las causas son complejas e interrelacionadas. Una posible explicación sería que las niñas, bajo la influencia del rol de género que nuestra sociedad les ha otorgado y que van adquiriendo conforme crecen, tienden a ocultar sus altas capacidades, puesto que en las mujeres son otro tipo de cualidades las que se premian y toman en consideración. Además, esta influencia de la socialización de género estaría incidiendo en sus expectativas y la percepción sobre sus propias capacidades. En este sentido, algunos autores han acuñado, indistintamente, términos en sus investigaciones como síndrome del impostor, complejo de cenicienta, síndrome de la perfección o síndrome de la abeja reina (Arendar, 2014; Clance e Imes, 1978; Dowling, 2014; Jiménez, 2014; Kerr, 1997; Pérez, 2002; Trillo, 2012) para tratar de explicar y definir las diferencias en variables socioemocionales, caso de la autoestima, que pueden Ilegar a minimizar sensiblemente el desempeño intelectual de las mujeres más capaces. En esta investigación tomamos la autoestima como variable principal de análisis para intentar explicar esta discrepancia entre sexos.

Una baja autoestima podría ser la causa de una autoexigencia desmedida, sentimiento de culpa, ansiedad, depresión, insatisfacción personal y frustración, entre otras, lo que podría estar dificultando su avance académico y profesional. Por otro lado, si hablamos de altas capacidades, hay que tener en cuenta que en la actualidad se ha superado la limitación de considerar el coeficiente intelectual como único ele- 
mento de identificación. De este modo, constructos y variables de la personalidad, como es el caso de la autoestima, adquieren gran relevancia y protagonismo en el estudio de las altas capacidades en toda su amplitud, cuestión posiblemente más incisiva en el caso concreto de la población femenina.

La autoestima se define como la imagen mental que el sujeto construye sobre sí mismo y cómo se siente y se desenvuelve en el entorno en función de la misma, por lo que no es fácil reconocerla ni aislarla del resto de estados y percepciones mentales. Su carga emocional y afectiva le otorga un carácter personal y subjetivo, lo que deriva en dificultades y una elevada complejidad en su abordaje. Según Arendar (2014, p. 395), la autoestima se definiría como "el grado de confianza que tenemos en nuestros propios recursos, el grado de aceptabilidad y valor afectivo que nos atribuimos y fundamentalmente un modo equilibrado de apreciar nuestras virtudes y defectos y/o limitaciones". Esta valoración, sea positiva o negativa, va a afectar inevitablemente a la forma en la que nos relacionamos y desenvolvemos en los distintos ámbitos de nuestra vida: familiar, social, académico y/o profesional.

\section{Método}

En este artículo se desarrolla una investigación cuantitativa con carácter experimental y descriptivo. Se han realizado análisis de resultados descriptivos y diferenciales y se han extraído las conclusiones más importantes, incluyendo limitaciones e implicaciones para futuras investigaciones y para la práctica educativa.

Se plantea como objetivo principal de la misma el analizar las diferencias entre sexos de acuerdo a la autoestima, prestando una especial atención al alumnado con altas capacidades intelectuales. De esta forma, se busca abrir un cauce en la justificación de por qué existen desigualdades en educación según el sexo de los individuos. En nuestro sistema educativo aparecen diferencias en el desempeño académico y en el diagnóstico de altas capacidades entre niños y niñas, tradicionalmente acuñadas no por cualidades naturales ni por diferencias biológicas en cuanto a capacidades cognitivas sino más bien a roles de género que ambos desempeñan, siendo las propias perspectivas y la autoconfianza las que juegan un papel crucial.

El punto de partida de nuestra investigación es que existen menos alumnas diagnosticadas con altas capacidades que alumnos, tal y como se ha mostrado en la anterior Tabla 1, 55,39\% de hombres frente a 44,61\% de mujeres, no existiendo diferencias probadas en la inteligencia en función del sexo, tal y como se ha señalado en el apartado anterior. Según toda la bibliografía estudiada, la hipótesis de partida sería que las niñas tendrían una autoestima más baja que sus pares varones, lo que influiría en su rendimiento académico y en contar con menores cifras de diagnóstico.

Es por esto que el objetivo de este trabajo ha consistido en llevar a cabo una evaluación de la autoestima en una muestra de alumnos y alumnas de los cursos $4^{\circ}$, $5^{\circ}$ y $6^{\circ}$ de Educación Primaria, para después comparar los resultados en función de distintas variables moduladoras, siendo el sexo de los sujetos la que toma un mayor interés para la investigación. 


\section{Muestra}

La muestra ha sido seleccionada de estos niveles educativos mediante un muestreo probabilístico de tipo aleatorio. En total, han sido seleccionados 118 alumnos de diferentes centros escolares de Educación Infantil y Primaria repartidos de la siguiente manera tal y como puede observarse en la Tabla 2.

Tabla 2. Características de la muestra en función de las variables moduladoras

\begin{tabular}{lccc}
\hline \multirow{2}{*}{ Sexo } & Hombre & 67 & 56,8 \\
\cline { 2 - 4 } Altas capacidades detectadas & Mujer & 51 & 43,2 \\
\hline \multirow{3}{*}{ Rendimiento académico } & Sí & 21 & 82,2 \\
\cline { 2 - 4 } & No & 97 & 17,8 \\
\hline \multirow{3}{*}{ Edad } & Alto & 40 & 33,9 \\
\cline { 2 - 4 } & Medio & 50 & 42,4 \\
\cline { 2 - 4 } & Bajo & 28 & 23,7 \\
\hline \multirow{3}{*}{ Curso } & 9 & 25 & 21,2 \\
\cline { 2 - 4 } & 10 & 36 & 30,5 \\
\cline { 2 - 4 } & 11 & 34 & 28,8 \\
\cline { 2 - 4 } & 12 & 40 & 33,9 \\
\hline
\end{tabular}

Fuente: Elaboración propia.

\section{Variables}

En cuanto a las variables estudiadas, podemos diferenciar entre variables de constructo y variables moduladoras. En relación a las variables de constructo, se ha tomado como principal la autoestima, entendida como la valoración personal, positiva o negativa, de uno mismo y de su propia imagen mental.

Por otro lado, las variables moduladoras tenidas en cuenta han sido las siguientes:

- Sexo, donde distinguimos entre Hombre y Mujer. Se ha hecho un análisis estadístico exhaustivo al tratarse de la variable de mayor interés para los objetivos de esta investigación.

- Altas capacidades intelectuales detectadas, distinguiendo en la muestra los sujetos que tienen altas capacidades diagnosticadas.

- Rendimiento académico, cuantificándose en rendimiento Alto (calificaciones medias de Sobresaliente), Medio (calificaciones medias de Notable y Bien) y Bajo (calificaciones medias de Suficiente e Insuficiente). Esta variable ha sido seleccionada a fecha de junio de 2018 por medio de las calificaciones medias del alumnado en el conjunto de las áreas curriculares. 
- Edad, usando la edad cronológica de los sujetos en el momento del estudio, que va desde los 9 a los 12 años.

- Curso, diferenciando entre $4^{\circ}, 5^{\circ}$ y $6^{\circ}$ curso de Educación Primaria.

Estas variables quedan definidas para la muestra seleccionada en la Tabla 2.

\section{Instrumento}

El instrumento empleado para evaluar la autoestima ha sido la Escala de Autoestima de Rosemberg. Concretamente se ha empleado la escala traducida incluida en la versión en castellano del libro de Rosenberg La autoimagen del adolescente y la sociedad (1973). Además, se ha tomado una modificación en la redacción para su adaptación a un lenguaje sensible al género (Vázquez, Jiménez, y Vázquez-Morejón, 2004)

Este instrumento es ampliamente utilizado para evaluar la autoestima, considerándose como un buen predictor para la medida de este constructo (Atienza, Moreno, y Balaguer, 2000). Su sencillez y rápida y fácil aplicación, unida a sus propiedades psicométricas, vienen a justificar su uso frecuente con población escolar de diversas etapas educativas, Educación Primaria, Educación Secundaria y Enseñanza Universitaria. Numerosos estudios de distintos países y contextos respaldan la validez y fiabilidad de la escala (caso de Góngora, y Casullo, 2009). El índice de fiabilidad del instrumento Alpha de Cronbach oscila entre ,82 y ,86 según distintas investigaciones, ejemplos señalados a continuación, y la versión española utilizada para esta investigación ha sido validada en población adolescente (Atienza, Moreno, y Balaguer, 2000) y universitaria (Reina, Oliva, y Parra, 2010).

Este cuestionario consta de 10 ítems, de los cuales 5 están redactados de forma positiva y 5 en negativo con vistas a controlar el posible sesgo de responder a todos ellos afirmativamente. La escala de respuesta es tipo Likert de 4 opciones (Muy de acuerdo, De acuerdo, En desacuerdo y Muy en desacuerdo) y, por tanto, las puntuaciones oscilan entre 10 a 40 puntos.

\section{Procedimiento}

Tras solicitar por escrito a la dirección de varios centros educativos la participación en este estudio, se solicitó la autorización por escrito a las familias de los niños y niñas cuyos centros y docentes estaban interesados en formar parte de la investigación. La selección de los centros estaba encaminada a la elección de escuelas que tuvieran escolarizados en sus aulas alumnado de altas capacidades intelectuales.

Una vez seleccionada la muestra, se procedió a administrar el cuestionario de manera colectiva para cada curso participante. Se pidió a los tutores que indicasen para cada alumno el nivel de rendimiento académico medio en función de sus calificaciones a fecha de junio de 2018, tal y como se ha descrito en el apartado anterior. Para el tratamiento de los datos se ha utilizado el programa estadístico IBM SPSS Statistics en su versión 22.0. 


\section{Resultados}

Tras la corrección de los cuestionarios empleados para evaluar la autoestima en la muestra participante, se ha procedido al tratamiento estadístico de los datos. Se especifican los resultados para la variable autoestima en función de otras variables moduladoras como sexo, altas capacidades detectadas, rendimiento académico, edad y curso escolar.

\section{Resultados obtenidos para la variable autoestima}

A continuación, se relacionan los resultados de la Escala de Autoestima de Rosenberg, recordamos que puntúa de 10 a 40 puntos. En la Tabla 3 se señala la frecuencia de cada una de las puntuaciones obtenidas por los sujetos, así como el porcentaje correspondiente.

En esta Tabla 3 se observa que la frecuencia aumenta a partir de P30 para volver a descender a partir de P35, siendo considerablemente más frecuentes las puntuaciones P31 y P34. No existen resultados de P10 a P13, P18, P19 y P22. Por otra parte, la media de todas las puntuaciones ha sido de 31,14 con una desviación típica de 5,50.

Tabla 3. Frecuencia y porcentaje para la variable autoestima

\begin{tabular}{lcc}
\hline Puntuación (P) & f & \% \\
\hline P14 & 1 & 0,8 \\
\hline P15 & 1 & 0,8 \\
\hline P16 & 2 & 1,7 \\
\hline P17 & 1 & 0,8 \\
\hline P20 & 1 & 0,8 \\
\hline P21 & 2 & 1,7 \\
\hline P23 & 2 & 1,7 \\
\hline P25 & 3 & 2,5 \\
\hline P26 & 5 & 4,2 \\
\hline P27 & 4 & 3,4 \\
\hline P28 & 2 & 1,7 \\
\hline P29 & 4 & 3,4 \\
\hline P31 & 6 & 5,1 \\
\hline P32 & 10 & 8,5 \\
\hline P33 & 13 & 11,0 \\
\hline P34 & 8 & 6,8 \\
\hline P35 & 9 & 7,6 \\
\hline P36 & 13 & 11,0 \\
\hline P37 & 6 & 5,1 \\
\hline P38 & 7 & 5,9 \\
\hline P39 & 6 & 5,1 \\
\hline P40 & 4 & 3,4 \\
\hline Total & 7 & 5,9 \\
\hline Fuen & 1 & 0,8 \\
\hline
\end{tabular}

Fuente: Elaboración propia. 
Para un análisis más exhaustivo de esta variable se han establecido tres niveles por intervalos de puntuación. De este modo, el nivel 1 ha sido considerado como el bajo, el nivel 2 como el medio y el nivel 3 como el alto. En la Tabla 4, se especifican los intervalos de cada nivel, así como la frecuencia y el porcentaje correspondiente.

Tabla 4. Frecuencias y porcentajes para cada nivel para la variable autoestima

\begin{tabular}{lccc}
\hline Niveles (N) & Intervalo & f & \% \\
\hline N1 & $10-19$ & 5 & 4,2 \\
N2 & $20-29$ & 29 & 24,6 \\
N3 & $30-40$ & 84 & 71,2 \\
\hline
\end{tabular}

Fuente: Elaboración propia.

Estos resultados por niveles muestran que la autoestima de los sujetos estudiados se encuentra en su mayoría en el nivel alto, pues el $71,2 \%$ de los sujetos han obtenido puntuaciones que se encuentran en dicho nivel, siendo solo de un $4,2 \%$ de la muestra la que se encuentra en el nivel más bajo de autoestima. La media de las puntuaciones según estos tres niveles es 2,67, con una desviación típica de ,56.

\section{Resultados en función del sexo}

En este apartado nos centramos en analizar en profundidad los resultados de la variable autoestima en función del sexo de la muestra participante. Se trata de la variable moduladora de mayor interés para esta investigación, importancia también observada en otros estudios sobre autoestima (Pastor, Balaguer, y García-Merita, 2003; Zeidner y Shani-Zinovich, 2015).

En primer lugar, se analizan los resultados para la variable sexo en función de los 3 niveles de autoestima establecidos. Indicar que la media de las puntuaciones alcanzadas por los alumnos de sexo masculino ha sido de 2,76, desviación típica de ,43, y para el sexo femenino de 2,55, desviación típica de ,67. Para cada nivel de forma específica, nos encontramos especificado en la Tabla 5 sus frecuencias y porcentajes.

Tabla 5. Frecuencias y porcentajes para la variable sexo según cada nivel definido para la variable autoestima

\begin{tabular}{lrrrr}
\hline Niveles (N) & \multicolumn{2}{c}{ Hombre } & \multicolumn{3}{c}{ Mujer } \\
\hline & \multicolumn{1}{c}{$\mathbf{f}$} & \% & f & \% \\
\cline { 2 - 5 } N1 & 0 & 0 & 5 & 4,23 \\
N2 & 16 & 13,56 & 13 & 11,02 \\
N3 & 51 & 43,22 & 33 & 27,97 \\
\hline
\end{tabular}

Fuente: Elaboración propia.

En esta tabla, se observa que en el nivel 1, el más bajo, aparece exclusivamente alumnado de sexo femenino, 5 casos. En los niveles 2 o medio, los porcentajes son de $13,56 \%$ para el sexo masculino y $11,02 \%$ para el femenino. En el último de los 
niveles, el 3 o alto, se observa mayor porcentaje del sexo masculino en comparación con el femenino, 43,22\% frente a 27,97\% respectivamente. Aunque parte de estas diferencias podrían deberse a la desigual muestra participante según el sexo, 67 hombres frente a 51 mujeres, los resultados obtenidos en el nivel más bajo justifican la necesidad de profundizar en el análisis estadístico para estas variables.

Para ello, se realizan en primer lugar las pruebas Chi-cuadrado, resultados reflejados en la Tabla 6 . Tras obtener significatividad en las mismas, resulta necesario continuar con el análisis de la relación entre sexo y autoestima realizando una prueba de comparación de medias (ANOVA), cuyos resultados aparecen en la Tabla 7. El resultado de la Eta ha sido ,19 y de la Eta cuadrado de ,04. El índice de correlación entre ambas variables ha sido de -,19, con una significatividad de ,04. Analizando el conjunto de los resultados, se observan puntuaciones estadísticamente significativas entre sexo y autoestima, quedando demostrada la obtención de puntuaciones más bajas en autoestima por parte del sexo femenino.

Tabla 6. Pruebas Chi-cuadrado para la variable sexo según cada nivel definido para la variable autoestima

\begin{tabular}{llcc}
\hline & Valor & gl & Sig. \\
\hline $\begin{array}{l}\text { Chi-cuadrado } \\
\text { de Pearson }\end{array}$ & $7,13^{\mathrm{a}}$ & 2 &, 02 \\
\hline $\begin{array}{l}\text { Razón de vero- } \\
\text { similitudes }\end{array}$ & 8,95 & 2 &, 01 \\
\hline $\begin{array}{l}\text { Asociación lineal } \\
\text { por lineal }\end{array}$ & 4,22 & 1 &, 04 \\
\hline
\end{tabular}

Fuente: Elaboración propia.

Tabla 7. ANOVA entre sexo y autoestima

\begin{tabular}{lcrccc}
\hline & $\begin{array}{c}\text { Suma de } \\
\text { cuadrados }\end{array}$ & gl & Media cuadrática & F & Sig. \\
\hline Inter-grupos & 1,30 & 1 & 1,30 & 4,34 &, 04 \\
(Combinadas) & 34,81 & 116 &, 30 & & \\
Intra-grupos & 36,11 & 117 & & & \\
Total & & & & & \\
\hline
\end{tabular}

Fuente: Elaboración propia.

\section{Resultados en función del resto de variables moduladoras}

El resto de variables utilizadas en la investigación han sido las siguientes: alta capacidad detectada, rendimiento académico, edad y curso. A continuación, se exponen los resultados para cada una de ellas.

En primer lugar, dentro de la variable alta capacidad detectada, definida como sí o no, aparecen en la Tabla 8 los resultados para cada nivel de autoestima. Al analizar 
los resultados para la variable alta capacidad detectada según los 3 niveles de autoestima fijados, la media de las puntuaciones logradas por los escolares que no son de altas capacidades es de 2,77, desviación típica de ,42, mientras que los que sí están detectados de altas capacidades ha sido de 2,19, desviación típica de ,81. En la Tabla 8 se observan las frecuencias y porcentajes para cada nivel de autoestima.

Tabla 8. Frecuencias y porcentajes para la variable alta capacidad detectada según cada nivel definido para la variable autoestima

\begin{tabular}{lcccc}
\hline \multicolumn{1}{c}{ Niveles (N) } & \multicolumn{2}{c}{ Sí } & \multicolumn{2}{c}{ No } \\
\hline & $\mathbf{f}$ & $\mathbf{\%}$ & $\mathbf{f}$ & $\mathbf{\%}$ \\
\cline { 2 - 5 } N1 & 5 & 4,24 & 0 & 0 \\
\hline N2 & 7 & 5,93 & 22 & 18,64 \\
\hline N3 & 9 & 7,63 & 75 & 63,56 \\
\hline
\end{tabular}

Fuente: Elaboración propia.

En esta tabla, Ilama especialmente la atención que los 5 escolares situados en el nivel 1 de autoestima tienen altas capacidades intelectuales y sexo femenino. Un $63,56 \%$ de los alumnos que no tienen diagnóstico de altas capacidades, de un total de un $82,20 \%$, se ubican en el nivel más elevado de autoestima. Las diferencias en porcentaje entre alumnos con y sin altas capacidades también vienen derivadas de la desigual muestra participante. Las pruebas Chi-cuadrado mostradas en la Tabla 9 reflejan resultados estadísticamente significativos (nivel de significatividad de ,00). De acuerdo a la comparación de medias (ANOVA), Tabla 10, aparecen niveles de significación de ,00. El resultado de la Eta ha sido ,40 y de la Eta cuadrado de ,16. El índice de correlación entre ambas variables ha sido de -,40, con una significatividad de ,00. Por otro lado, si correlacionamos niveles de autoestima, sexo y alta capacidad detectada, el índice obtenido es de -,19 con un nivel de significación de ,04, demostrando la significatividad entre estas variables, sobre todo en la relación entre autoestima y alta capacidad femenina.

Tabla 9. Pruebas Chi-cuadrado para la variable alta capacidad detectada según cada nivel definido para la variable autoestima

\begin{tabular}{lccc}
\hline & Valor & gl & Sig. \\
\hline Chi-cuadrado de Pearson & $26,77^{\mathrm{a}}$ & 2 &, 00 \\
\hline Razón de verosimilitudes & 21,26 & 2 &, 00 \\
\hline Asociación lineal por lineal & 18,99 & 1 &, 00 \\
\hline
\end{tabular}

Fuente: Elaboración propia.

La siguiente variable de análisis es el rendimiento académico. Para esta variable, de acuerdo a las pruebas Chi-cuadrado, ANOVA e índices de correlación no aparecen diferencias estadísticamente significativas. Por otro lado, aparecen resultados relevantes tras observarse que existe alumnado de rendimiento elevado, sexo femenino y alta capacidad detectada ubicado en el nivel más bajo de autoestima, 3 alumnas, obteniéndose una correlación de -,20 y un nivel de significación de ,03. 
Una vez analizados los resultados para la variable de rendimiento académico, se pasan a especificar los resultados en función de la edad y curso de los alumnos participantes. Para estas variables, no se observan diferencias estadísticamente significativas, por lo que se podría afirmar que en este intervalo de edad, 9-12 años, y para la etapa de Educación Primaria, todavía no aparecen diferencias en cuanto a los niveles de autoestima del alumnado, incidirían otras variables tal y como ha quedado demostrado anteriormente.

\section{Conclusión}

Una vez analizados los resultados del estudio se ha comprobado que los sujetos evaluados tienen en general unos niveles altos de autoestima, pero se confirma que las niñas presentan niveles de autoestima más bajos que los niños. También se ha observado cómo las alumnas con altas capacidades muestran niveles de autoestima más bajos que los alcanzados por sus iguales de sexo masculino, siendo muy relevante que los 5 escolares situados en el nivel 1 de autoestima son mujeres y con diagnóstico de altas capacidades intelectuales.

Además de esta relación comprobada entre el constructo evaluado con el sexo y la alta capacidad detectada, también existe interacción con el rendimiento académico, ya que aparece alumnado de rendimiento elevado que puntúa más bajo en autoestima, coincidiendo que son alumnas con altas capacidades intelectuales. De este modo, se sigue la línea argumental de la literatura en la que se ha basado esta investigación y se confirma la hipótesis de trabajo planteada, las niñas, por lo general, pueden manifestar niveles de autoestima más bajos que los niños, lo que podría influir en su rendimiento académico futuro y conllevar invisibilidad de su alta capacidad en edad escolar, aspecto interesante para profundizar en futuras investigaciones. Sin embargo, no se han hallado resultados significativos entre la variable autoestima y el resto de variables analizadas, edad y curso.

Y dado que los resultados en función de la edad no han arrojado cifras significativas, para futuras investigaciones sería conveniente llevar a cabo un análisis longitudinal que evalúe estas variables, pues según la literatura aportada (caso de: Atienza, Moreno, y Balaguer, 2000; Reina, Oliva, y Parra, 2010), en edades tempranas no existiría brecha en cuanto a aptitudes y autoestima, sino que esta iría apareciendo conforme los niños y niñas van acercándose a la adolescencia y juventud. De este modo se podría comprobar si existen variaciones en la autoestima conforme la edad avanza.

Además, se considera necesario abordar nuevos trabajos que analicen la posible relación de la discriminación que la mujer ha venido sufriendo históricamente en todos los ámbitos y la socialización de género que todavía hoy "rige" nuestra cultura, focalizando la atención en las mujeres con altas capacidades intelectuales y prestando una especial consideración al papel de la autoestima en los procesos de construcción de la personalidad.

Para ello, cabe plantearse las implicaciones y responsabilidades que deben de tomar los dos agentes de socialización más importantes para los niños y niñas: la familia y la escuela. En este sentido, se ha avanzado mucho hasta nuestros días y las 
Ciencias de la Educación han contribuido decisivamente en progresos decisivos conseguidos. Pero aún hoy persisten obstáculos en los ámbitos educativo y profesional para la mujer, la desigualdad de género continúa siendo una cuestión a debate que no debe ser ignorada por el sistema educativo, queda camino por recorrer en múltiples campos como: coeducación, autoestima, estereotipos y prejuicios, formación docente, altas capacidades intelectuales, itinerarios académicos, entre otros.

De otra parte, es también fundamental el rol que tiene la familia en los procesos educativos, y en la construcción de una autoestima positiva. Por un lado, las pautas de educación transmitidas desde el entorno familiar podrían estar reproduciendo las desigualdades de género y los estereotipos existentes en parte de los ámbitos de nuestra sociedad. Es por ello que se debe ser consciente de la importancia que puede tener la familia en la construcción de la autoestima del sujeto, variable con incidencia determinante en los porcesos educativos tal y como ha quedado de manifiesto.

Para terminar, hemos de admitir que la solución al problema abordado todavía se vislumbra lejana por su complejidad y la multitud de dimensiones que abarca, pero si todos los agentes sociales aunamos esfuerzos y luchamos por una igualdad de oportunidades real, quizá algún día la mujer altamente capaz salga de su caparazón y deje de ser invisible a ojos de una sociedad que tanto necesita de ella. Y ese día, estudios como éste dejarán de ser necesarios.

\section{REFERENCIAS BIBLIOGRÁFICAS}

Arendar, R. (2014). La autoestima y sus vicisitudes. Clínica e Investigación Relacional, 8(3), 394-402.

Atienza, F. L., Moreno, Y. y Balaguer, I. (2000). Análisis de la dimensionalidad de la Escala de Autoestima de Rosenberg en una muestra de adolescentes valencianos. Revista de Psicología Universitas Tarraconensis, 23(1-2), 29-42.

Baeza, A. y Lamadrid, S. (2018). Trayectorias educativas según género. Lo invisible para la política educativa chilena. Revista de Investigación Educativa, 36(2), 471-490.

Bian, L., Leslie, S. J. y Cimpian, A. (2017). Gender stereotypes about intellectual ability emerge early and influence children's interests. Science, 355(6323), 389-391.

Campo, M. (2016). Regulación emocional y habilidades sociales en niños con altas capacidades intelectuales. Tesis de doctorado para la obtención del título de Doctora en Psicología, Universidad Complutense de Madrid.

Choi, A. y Calero, J. (2013). Determinantes del riesgo de fracaso escolar en España en PISA 2009 y propuestas de reforma. Revista de Educación, 362, 562-593.

Clance, P. L. e Imes, S. (1978). The Imposter Phenomenon in High Achieving Women: Dynamics and Therapeutic Intervention. Psychotherapy, Theory Research and Practice, 15(3), 241-247.

Colás, P. y Villaciervos, P. (2007). La interiorización de los estereotipos de género en jóvenes y adolescentes. Revista de Investigación Educativa, 27(1), 35-58. 
Dowling, C. (2014). El complejo de Cenicienta: El miedo de las mujeres a la independencia. Madrid: Debolsillo.

Elices, J., Riveras, F., González, C. y Crespo, M. (1990). El rendimiento escolar en función del sexo al inicio de la EGB. Revista Interuniversitaria de Formación del Profesorado, 8, 123-132.

Garaigordobil M. y Amigo, R. (2010). Diferencias de género y relaciones con factores psicomotrices, conductuales y emocionales en niños de 5 años. Interdisciplinaria, $27(2), 229-245$.

García, R., Sala, A., Rodríguez, E. y Sabuco, A. (2013). Formación inicial del profesorado sobre género y coeducación: impactos metacognitivos de la inclusión curricular transversal sobre sexismo y homofobia. Profesorado, Revista de currículum y formación del profesorado, 17(1), 269-270.

Góngora, V. y Casullo, M. ${ }^{a}$ M. (2009). Validación de la escala de autoestima de Rosenberg en población general y en población clínica de la Ciudad de Buenos Aires. Revista Iberoamericana de Diagnóstico y Evaluación Psicológica, 27(1), 179-174.

González-Palencia, R. y Jiménez, C. (2016). La brecha de género en la educación tecnológica. Ensaio: Avaliação e Políticas Públicas em Educação, 24(92), 743-771.

Instituto Nacional de Evaluación Educativa (2013). PISA 2012: Informe Español. Volumen I: Resultados y contexto. Madrid: Ministerio de Educación, Cultura y Deporte. Recuperado de http://www.mecd.gob.es/dctm/inee/internacional/pisa2012/ pisa2012lineavolumeni.pdf?documentld=0901e72b81786310.

Jiménez, C. (2014). El desarrollo del talento: educación y alta capacidad. Madrid: Universidad Nacional de Educación a Distancia.

Jiménez, C., Murga, M. A., Gil, J. A., Téllez, J. A. y Paz, M. (2010). Hacia un modelo sociocultural explicativo del alto rendimiento y la alta capacidad: ámbito académico y capacidades personales. Revista Educación XXI, 13(1), 125-153.

Kerr, B. (1997). Smart Girls. A new psychology of girls, women, and giftedness. Scottsdale, AZ, United States: Gifted Psychology Press.

Kreger, L. (1999). Todo empezó con Leta Hollingword: historia de la superdotación en las mujeres. En J. Ellis y J. Willinsky, Niñas, Mujeres y Superdotación. Un desafío a la discriminación educativa de las mujeres (pp. 39-50). Madrid, España: Narcea.

Llor, L., Ferrando, M., Ferrandiz, C., Hernández, D., Sainz, M., Prieto, M. D. y Fernández, M.C. (2012). Inteligencias Múltiples y Alta Habilidad. Aula Abierta, 40(1), 27-38.

Ministerio de Educación, Cultura y Deporte (2016a). PISA 2015. Informe español. Boletín de Educación, 51, 1-4. Recuperado de http://www.mecd.gob.es/dctm/inee/ boletines/educainee51 provokk.pdf?documentld=0901e72b8228c0b9.

Ministerio de Educación, Cultura y Deporte (2016b). PISA 2015. Programa para la Evaluación Internacional de los Alumnos. Informe español. Madrid: Ministerio 
de Educación, Cultura y Deporte. Recuperado de http://www.mecd.gob.es/dctm/ inee/internacional/pisa-2015/pisa2015preliminarok.pdf?documentld=0901e72b8 228b93c.

Ministerio de Educación, Cultura y Deporte (2018). Estadísticas no universitarias. Recuperado de http://www.mecd.gob.es/servicios-al-ciudadano-mecd/estadisticas/ educacion/no-universitaria.html

Organización para la Cooperación y el Desarrollo Económicos (2012). PISA. The $A B C$ of Gender Equality in Education: Aptitude, Behaviour, Confidence. Recuperado de https://www.oecd-ilibrary.org/education/the-abc-of-gender-equality-ineducation_9789264229945-en.

Organización para la Cooperación y el Desarrollo Económicos (2014). PISA 2012. Programa para la evaluación internacional de los alumnos. Informe Español. Resultados y contexto. Recuperado de https://www.mecd.gob.es/inee/evaluacionesinternacionales/pisa/pisa-2012.html.

Organización para la Cooperación y el Desarrollo Económicos (2016). PISA 2015. Programa para la evaluación internacional de los alumnos. Informe Español. Recuperado de https://www.mecd.gob.es/inee/evaluaciones-internacionales/pisa/pisa-2015.html.

Pasarín, M., Feijoo, M., Díaz, O. y Rodríguez, L. (2004). Evaluación del talento matemático en Educación Secundaria. Revista internacional Faisca de Altas Capacidades, 11, 83-102.

Pastor, Y., Balaguer, I. y García-Merita, M. L. (2003). El autoconcepto y la autoestima en la adolescencia media: análisis diferencial por curso y género. Revista de Psicología Social, 18(2), 141-159.

Peña del Agua, A. M. (2002). Superdotación: factores culturales y barreras sociales. Revista de Educación de la Universidad de Huelva, 4, 261-269.

Pérez, L. (2002). Mujeres superdotadas y sociedad: del burka al síndrome de abeja reina. FAISCA, Revista de Altas Capacidades, 9, 35-55.

Pérez, L. y Domínguez, P. (2000). Superdotación y adolescencia. Características y necesidades en la Comunidad de Madrid. Madrid: Consejería de Educación.

Reina, M. C., Oliva, A. y Parra, A. (2010). Percepciones de autoevaluación: Autoestima, autoeficacia y satisfacción vital en la adolescencia. Psychology, Society, \& Education, 2(1), 55-69.

Rosenberg, M. (1973). La autoimagen del adolescente y la sociedad. Buenos Aires, Argentina: Paidós.

Saavedra, F. J., Bascón, M. J., Prados, M. M. y Sabuco, A. (2013). Indicadores y criterios de calidad de buenas prácticas coeducativas. Una propuesta innovadora. Profesorado, revista de currículum y formación del profesorado, 17(1), 201-220.

Suberviola, I. (2012). Coeducación: un derecho y un deber del profesorado. Revista Electrónica Interuniversitaria de Formación del Profesorado, 15(3), 59-67. 
Trillo, M. C. (2012). Alta capacidad y género. Factores diferenciadores cognitivos y de personalidad en niños y niñas de alta capacidad. Tesis de doctorado para la obtención del título de Doctora en Educación, Universidad de Córdoba.

Vázquez, A. J., Jiménez, R. y Vázquez-Morejón, R. (2004). Escala de autoestima de Rosenberg: Fiabilidad y validez en población clínica española. Apuntes de Psicología, 22(2), 247-255.

Zeidner, M. y Shani-Zinovich, I. (2015). A comparison of multiple facets of self-concept in gifted vs. non-identified Israeli students. High Ability Studies, 26(2), 211 226. 